\title{
Unexpected behavior of irradiated spider silk links conformational freedom to mechanical performance
}

\author{
G. Belén Perea, Concepción Solanas, Gustavo R. Plaza, Gustavo V. Guinea, \\ Inmaculada Jorge, Jesús Vázquez, Jorge M. Pérez Mateos, Núria Marí-Buyé, \\ Manuel Elices' and José Pérez-Rigueiro
}

\begin{abstract}
Silk fibers from Argiope trifasciata and Nephila inaurata orb-web weaving spiders were UV irradiated to modify the molecular weight of the constituent proteins. Fibers were characterized either as forcibly silked or after being subjected to maximum supercontraction. The effect of irradiation on supercontraction was also studied, both in terms of the percentage of supercontraction and the tensile properties exhibited by irradiated and subsequently supercontracted fibers. The effects of UV exposure at the molecular level were assessed by polyacrylamide gel electrophoresis and mass spectrometry. It is shown that UV-irradiated fibers show a steady decrease in their main tensile parameters, most notably, tensile strength and strain. The combination of the mechanical and biochemical data suggests that the restricted conformational freedom of the proteins after UV irradiation is critical in the reduction of these properties. Consequently, an adequate topological organization of the protein chains emerges as a critical design principle in the performance of spider silk.
\end{abstract}

\section{Introduction}

Spider silk is one of the main representative topics in which the principles and applications of biomimetics ${ }^{1}$ will have to validate the large expectations that arise in this research area. However, understanding the unique properties of this material in terms of a few clear, concise and, especially, predictable ideas has proven to be extremely challenging. The most sophisticated attempts intended to unveil the subtle combination and interplay of protein sequences, processing, microstructure and properties, which endow the natural material with its outstanding features, ${ }^{2}$ have only led to the identification of some partial aspects of the material's behaviour.

In this regard, one of the most fruitful strategies appears to be the comparison of the microstructure and mechanical behaviour of silks spun by different spider lineages or, alternatively, of different silk types spun by a single species. This approach is based on the significant similarities and differences exhibited by the sequences of different spidroins ${ }^{3}$ that constitute silks with widely different biological functions. ${ }^{4}$ It was found that major ampullate gland silk spidroins of orb-web weaving spiders (Orbicularians) show the most complex organization among spider silks with the presence of four basic motifs: $-\mathrm{A}_{n^{-}}$, -GA-, -GGX- (where $\mathrm{X}$ stands for a small number of amino acids, such as tyrosine) and -GPG-. ${ }^{5}$ Other silks spun by different species and/or glands are characterized by the absence of at least one of these motifs. For instance, it was found that major ampullate gland silks of spider lineages different from Orbicularians do not present the motif -GPG-, ${ }^{6}$ which does not appear either in the minor ampullate gland silk of Orbicularians. ${ }^{7}$ In contrast, the flagelliform gland silk of Orbicularians presents a large content of the -GPG- motif, but the $-\mathrm{A}_{n}$ - motif is absent.

This strategy has allowed establishing correlations between the different primary structures of spidroins and the behaviour of the fibers. ${ }^{8-10}$ In particular, it was possible to propose definite roles for the different motifs with regard to the phenomenon of supercontraction. Supercontraction is a characteristic large shrinkage of major ampullate gland silk under high humidity conditions, ${ }^{11}$ which is absent in silks spun by other organisms. It was found that supercontraction is linked to the presence of the -GGX- motif, ${ }^{12}$ although the quantitative manifestation of the effect in terms of supercontracted length 
and strain at breaking is notably affected by the presence of the -GPG- motif. ${ }^{13,14}$

Unfortunately, spider silks display only a relatively small number of natural variants-such as major ampullate, minor ampullate and flagelliform silks-which severely limits the analytical power of this approach. Ideally, extension of this basic strategy would imply characterization of silk fibers previously subjected to physicochemical treatments that would modify their microstructure in a controlled way. Practical realization of this methodology, however, has proven to be a challenging problem due to (a) the requirement to develop controllable physicochemical treatments and (b) the need to characterize the subsequent changes induced in the fibers at the molecular level.

Several procedures intended to modify the microstructure of silks were proposed, including the use of crosslinking agents, ${ }^{15-17}$ heating ${ }^{18}$ and immersion in different solvents. ${ }^{19}$ In this regard, the analysis of supercontraction and related effects has allowed establishing a description of spider silk based on the existence of a double lattice of hydrogen bonds and elastomeric chains linked by protein nanocrystallites. ${ }^{20-22}$

UV irradiation has long been recognized as one of the major environmental conditions that degrade silk. Initial interest in the effect of UV light on silk was motivated by conservation problems of historic fabrics in museums and expositions. ${ }^{23}$ It was found that the changes induced by UV light on silk fibers included mass loss, ${ }^{23}$ yellowing due to the creation of new chromophores $^{24}$ and generation of lower weight molecules. ${ }^{25}$ Subsequent analysis of the tensile behaviour of irradiated fibers revealed a significant decrease in their mechanical properties in both silkworm ${ }^{26}$ and spider silk, ${ }^{27}$ despite the fact that fractographic analysis of the fibers did not reveal the presence of more defects after irradiation. ${ }^{28}$

There is, however, some controversy regarding the detailed effects exerted on the fibers by UV radiation. Thus, while the energy of UV photons might be sufficient to cause the breaking of covalent bonds in silk, ${ }^{29,30}$ as it has been found in other polymeric materials, ${ }^{31}$ the energy of the radicals is also high enough to create new crosslinks between protein chains. ${ }^{25}$ Additionally, there is also a debate on the nature of the amino acids that are primarily affected by UV radiation. Several studies propose that the main effect of radiation is restricted to a subset of amino acids including tyrosine, ${ }^{25,26}$ glycine and alanine $e^{24}$ and sulphur-containing residues. ${ }^{32}$ Alternatively, other studies suggest the creation of $\mathrm{C} \alpha$-centered radicals, which might expand the set of amino acids affected by irradiation.

At this point it should be noticed that UV light from natural sources is mainly composed of UV-A and UV-B photons with wavelengths in the range of $400-280 \mathrm{~nm}$, since $\mathrm{UV}-\mathrm{C}$ radiation of higher energy is filtered by the atmosphere. However, since the basic aim of this work is to take advantage of UV light in order to deepen our knowledge on the behavior of silk, we have irradiated the fibers with high energy UV-C light (wavelength $\lambda=254 \mathrm{~nm}$ ) to promote the physicochemical changes in the material. In this regard, preliminary studies performed in our group showed that longer wavelengths (365 nm and $312 \mathrm{~nm}$ ) had a significantly milder effect in the silk fibers.
Regardless of its detailed effect on silk, exposure to UV light implies the variation of the molecular weight of the spidroins and, consequently, the characterization of irradiated fibers provides a convenient approach to explore the correlations between the microstructure and properties of spider silk. Following this rationale, the present work studies the mechanical properties of UV irradiated spider silk fibers and the relationship between UV exposure and supercontraction. The characterization of silk proteins by gel electrophoresis and mass spectrometry was then used to relate the observed changes in the mechanical behaviour with the chemical modifications in the spidroins.

\section{Experimental section}

\section{A. Spider silk retrieval}

Major ampullate silk samples of the spiders Argiope trifasciata and Nephila inaurata were retrieved by forced silking techniques. Conventional forced silking on a rotating mandre ${ }^{33}$ was used to retrieve yarns of sufficient mass for the biochemical analysis. Monitored forced silking ${ }^{34}$ was used for retrieving the fibers used in the tensile tests, since it has been found that silk fibers spun at similar silking forces yield comparable tensile properties, ensuring sufficient reproducibility of the initial material. Both single fibers and yarns were mounted on perforated plastic frames $^{35}$ to facilitate manipulation during the subsequent experimental tests.

Individual fibers were analysed either in the initial forced silking (FS) ${ }^{36}$ state or after being subjected to maximum supercontraction. ${ }^{11}$ Maximum supercontraction (MS) ${ }^{21}$ proceeds by mounting the fiber in a device that allows reducing the length between its ends, so that the fiber remains slack before being submerged in water. Upon immersion the fiber shrinks (supercontracts) and, after being removed from water, is allowed to dry overnight. Maximum supercontraction is assessed by checking that the fiber is not pre-stressed before being mechanically tested. Supercontraction is quantified through the percentage of supercontraction, ${ }^{21}$ that is defined from the initial length of the fiber, $L_{0}$, and the length after maximum supercontraction, $L_{\mathrm{MS}}$, as $\% \mathrm{SC}=\left(L_{0}-L_{\mathrm{MS}}\right) / L_{0} . \mathrm{FS}$ and MS states correspond to the condition of maximum and minimum alignment of the protein chains, respectively. ${ }^{37}$

\section{B. Spider silk irradiation}

Samples were irradiated with $254 \mathrm{~nm}$ wavelength UV light using a VILBER LOURMAT 215LC lamp (Marne La Vallée, France) with the nominal intensity at $15 \mathrm{~cm}, I=1100 \mu \mathrm{W} \mathrm{cm}{ }^{-2}$. Samples were placed at a distance of $5.5 \mathrm{~cm}$ of the lamp for different times. Control samples were identically placed under the UV lamp, but covered by an opaque card to prevent direct irradiation, whilst taking into account other possible environmental effects such as heating.

\section{Tensile testing}

Tensile tests on individual fibers were performed in an INSTRON 4411 (Canton, Massachusetts, USA) mechanical testing machine. The initial length of the samples was $20 \mathrm{~mm}$ and tests were 
performed at a constant speed of $0.02 \mathrm{~mm} \mathrm{~s}^{-1}$. Forces were measured using an electronic balance (PRECISA XT 200, Milton Keynes, U.K. with a resolution of $1 \mu \mathrm{N}$ ) placed under the lower grip. The displacement of the crosshead was taken as a direct measurement of the deformation of the fiber, since it was estimated that the compliance of the fiber is at least 1000 times higher than that of the rest of the experimental setup. Tensile tests were performed either in air under nominal conditions of $25{ }^{\circ} \mathrm{C}$ and $35 \%$ relative humidity or in water. Tensile tests of MS fibers proceeded similarly, except for variations in the initial length which was no longer $20 \mathrm{~mm}$, but that of the fiber after maximum supercontraction. A detailed scheme of the testing procedure can be found elsewhere. ${ }^{37}$

The cross-sectional areas of the fibers were measured to convert forces into stresses. Both ends (approx. $5 \mathrm{~mm}$ long) of the samples tested were retrieved before tensile testing and subsequently metalized with gold for observation in a scanning electron microscope (JEOL 6300; Tokyo, Japan; observation conditions $V=10 \mathrm{kV}, I=0.06 \mathrm{nA})$. Three micrographs were obtained at different positions along each sample and three measurements were taken from each micrograph. The diameter of each tested sample was calculated as the average of all the measurements taken on both ends adjacent to that sample. The cross-sectional area was calculated from this diameter assuming a circular cross-section. ${ }^{38}$

True strain, $\varepsilon$, was calculated as $\mathrm{d} \varepsilon=\mathrm{d} L / L$, which leads to the expression $\varepsilon=\operatorname{Ln}(1+e)$, where $e$ is engineering strain defined as $e=\left(L-L_{0}\right) / L_{0}$, and $L_{0}$ and $L$ are the initial and instantaneous lengths of the sample, respectively. True stress was calculated as $\sigma=F / A$, where $A$ is the instantaneous area of the fiber. The instantaneous area of the fiber was calculated as $A=A_{0} L_{0} / L$ from the initial area, $A_{0}$, as obtained from the SEM measurements under the constant volume hypothesis. ${ }^{39}$

\section{Biochemical analysis: electrophoresis and quantitative amino acid analysis}

The biochemical study of silk included monitoring the evolution of the size of protein entities by gel electrophoresis and the analysis of the soluble and insoluble fractions after irradiation. The protein components of the gel bands were characterized by mass spectrometry.

Electrophoretic samples were prepared from approximately $0.5 \mathrm{mg}$ of either control or irradiated samples. All samples were treated with $0.5 \mathrm{~mL}$ of hexafluoroisopropanol (HFIP) for 3 days at $70{ }^{\circ} \mathrm{C}$ in an orbital shaker at $1300 \mathrm{rpm}$. It was observed that a fraction insoluble in HFIP appeared in irradiated samples. Samples were subsequently dried under vacuum and then resuspended in $120 \mu \mathrm{L}$ of electrophoretic loading buffer $-0.9 \mathrm{M}$ Tris-HCl, pH 8.45, 2\% (w/v) SDS, 10\% (v/v) glycerol, and $0.01 \%(\mathrm{w} / \mathrm{v})$ bromophenol blue containing $300 \mathrm{mM}$ dithiothreitol (DTT) and heat denaturalized. It was found that solubilisation of the irradiated samples was not complete, but the electrophoretic protein profiles for each condition were reproducible. Standard SDS-PAGE processes $(1.5 \mathrm{~mm}$-thick, $4 \%$ stacking gel and $5 \%$ resolving gel) were carried out using a MINI-PROTEAN TETRA system (Bio-Rad, Munich, Germany) using the resolving gel and run at constant voltage $(200 \mathrm{~V})$ according to the method described by Laemmli. ${ }^{40}$ SIGMAMARKER WIDE RANGE $6.5-200 \mathrm{kDa}$ (Sigma Aldrich, St. Louis, MO, U.S.A.) were used to determine the protein molecular masses. Gels were stained with Coomassie Brilliant Blue G250 and Sypro Ruby. In some cases, the previous protocol was modified to produce discontinuous gradient gels that allow taking into account the presence of protein fragments with a wide range of molecular weights. Discontinuous gradient gels were produced following the same steps as for the ordinary gels except that stripes with varying percentage of polyacrylamide were added consecutively. Four or five polyacrylamide stripes were typically prepared with a variation in the percentage of polyacrylamide ranging from $4 \%$ to $17 \%$.

The presence of an insoluble fraction after UV irradiation required the development of a new experimental procedure for its characterization and determination of the ratio between soluble and insoluble fractions. Approximately $0.5 \mathrm{mg}$ of control and irradiated samples were incubated in HFIP $(1 \mathrm{~mL}$ per $\mathrm{mg}$ of silk) for $210 \mathrm{~min}$ at $70^{\circ} \mathrm{C}$ in an orbital shaker at $1300 \mathrm{rpm}$. After evaporation of the solvent in air upon standing, samples were resuspended in the same volume of new HFIP $(1 \mathrm{~mL}$ per $\mathrm{mg}$ ) and stirred at $60{ }^{\circ} \mathrm{C}$ and $400 \mathrm{rpm}$ for $3 \mathrm{~h}$. The soluble fraction was then separated by suction using a needle $(0,3 \mathrm{~mm}$ ID) and deposited in a glass tube. The insoluble residue was next transferred to another glass tube by carefully using the tip of the needle, so that the possible contamination of the insoluble fraction with the remains of the soluble fraction in the original tube was reduced. Solvent was evaporated to dryness and both fractions were subjected to quantitative amino acid analysis. Briefly, after acid hydrolysis ( $\mathrm{HCl} 5.7 \mathrm{M}, 21 \mathrm{~h}, 110^{\circ} \mathrm{C}$, gas phase in a $\mathrm{N}_{2}$ atmosphere) of the proteins, chromatographic separation of the resulting amino acids was performed on an ion-exchange column with post-column derivation with ninhydrin (BIOCHROM 30 INSTRUMENT; Cambridge, UK), and imino and amino acids were detected at 440 and $570 \mathrm{~nm}$, respectively. L-norleucine was used as internal standard. For each sample, the entire procedure was performed in duplicates.

\section{E. Biochemical analysis: mass spectrometry}

Protein bands for mass spectrometry analysis were in-gel digested overnight at $37^{\circ} \mathrm{C}$ with $25 \mathrm{ng} \mu \mathrm{L}^{-1}$ quimotrypsin in $100 \mathrm{mM}$ Tris$\mathrm{HCl}$, pH 7.8 , containing $10 \mathrm{mM} \mathrm{CaCl}_{2}$. The resulting peptides were extracted by $1 \mathrm{~h}$ incubation in $100 \mathrm{mM}$ Tris-HCl, $\mathrm{pH} 7.8$. After acidification with 1\% trifluoroacetic acid (TFA), peptides were desalted onto a C18 zip-tip and dried. Isolated peptides were analysed by liquid chromatography tandem mass spectrometry (LC-MS/MS) using a Surveyor LC system coupled to an LTQ linear ion trap mass spectrometer (THERMO FINNIGAN, San José, CA), as described previously. ${ }^{41,42}$ Peptides were desalted on a reverse phase $(\mathrm{RP})$ precolumn $(0.32 \times 30 \mathrm{~mm})$ and on-line eluted on an RP column $(0.18 \times 150 \mathrm{~mm})$, operating at $2 \mu \mathrm{L} \mathrm{min}{ }^{-1}$ and 20 min gradient from $5 \%$ to $40 \%$ B [solvent A: $0.1 \%$ formic acid $(\mathrm{v} / \mathrm{v})$, solvent B: $0.1 \%$ formic acid $(\mathrm{v} / \mathrm{v})$ and $80 \%$ acetonitrile $(\mathrm{v} / \mathrm{v})]$.

LTQ was operated in a data-dependent MS/MS mode using the 10 most intense precursors detected in a survey scan from 400 to $1600 \mathrm{~m} / \mathrm{z}$. Protein identification in spidroin proteins 
using the uniprot FASTA database (release May 17, 2012; 366 entries; supplemented with porcine trypsin and human keratin) was carried out using the SEQUEST algorithm (Bioworks 3.2 package) as described in ref. 43 and 44 . The sequences provided by SEQUEST were verified by manual inspection of the corresponding fragmentation spectra.

\section{F. Recombinant protein fibers}

The experimental analysis performed on natural MAS fibers was repeated with recombinant silk fibers produced by the Nexia company. ${ }^{45,46}$ The recombinant protein is labelled as rcSp1 (recombinant spidroin 1) and includes repetitive motifs of sequence of the spidroin 1 protein of major ampullate silk from Nephila clavipes ${ }^{3}$ including the carboxy terminus. The molecular weight of the polypeptide is approximately $50 \mathrm{kDa}$. The processing details of the artificial fibers can be found elsewhere. ${ }^{46}$ Briefly, rcSp1 protein was purified to homogeneity from the milk of transgenic goat to $>95 \%$ purity. Spin dopes were prepared by adding HFIP and shaken until dissolved. Fibers were prepared using a 2-propanol coagulant bath and fibers were subjected to a steam atmosphere during postspinning drawing.

\section{Results}

\section{A. Mechanical behaviour of UV irradiated fibers}

The evolution of the mechanical behaviour of FS fibers spun by A. trifasciata and $N$. inaurata spiders upon UV irradiation is shown in Fig. 1. It is apparent that longer irradiation times lead to a steady decrease in the tensile strength and strain at breaking of the samples. Additionally, the overall stiffness of the fibers, which can be referenced to the values of stress reached at fixed strains, is observed to decrease with UV exposure. These trends had been previously reported from $A$. trifasciata UV irradiated samples, ${ }^{28}$ except for the decrease in the stiffness of the fibers, which was not considered relevant in the previous work, but can be clearly observed from the new data.

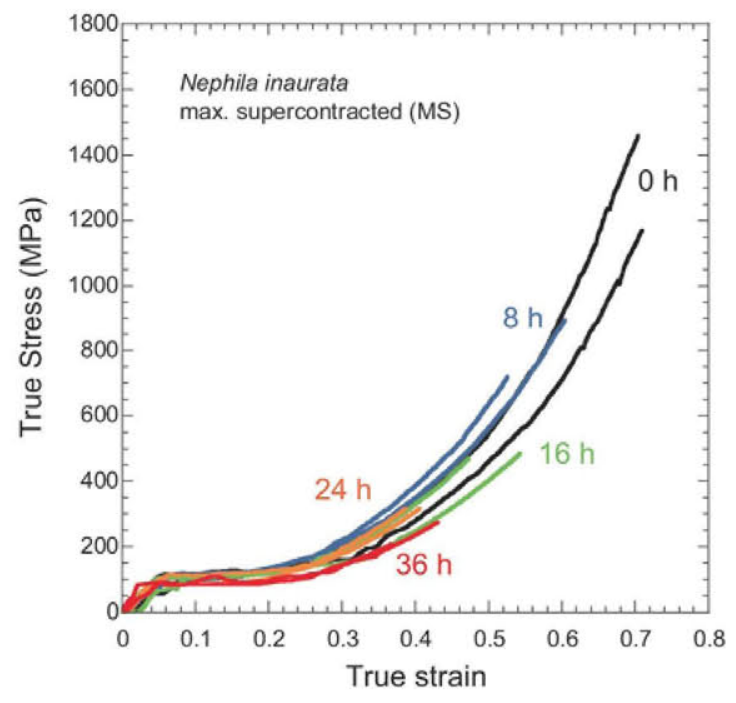

Fig. 2 True stress-true strain curves of Nephila inaurata MAS fibers subjected to maximum supercontraction and subsequent UV irradiation.

Tensile properties of Nephila inaurata FS fibers subjected to maximum supercontraction and subsequent UV irradiation are presented in Fig. 2. MS fibers exposed to UV light showed lower values of the tensile strength and strain at breaking compared with MS control samples, although the stress-strain curves of both control and treated MS samples concurred up to the breaking point of the latter. A similar behaviour was previously found in maximum supercontracted and UV irradiated Argiope trifasciata MAS fibers. ${ }^{28}$

The combined analysis of UV exposure and supercontraction has been extended in this work to include the characterization of UV irradiated samples that were subsequently subjected to supercontraction. Fig. 3 shows the evolution of the percentage of supercontraction (\%SC) with increasing irradiation times for A. trifasciata (Fig. 3a) and N. inaurata (Fig. 3b) silk. Increasing UV irradiation time leads to a steady decrease of the percentage of supercontraction in both materials, until a limiting value seems to be reached at least in $N$. inaurata fibers. Differences in the numerical values of \%SC found in both MAS silks are
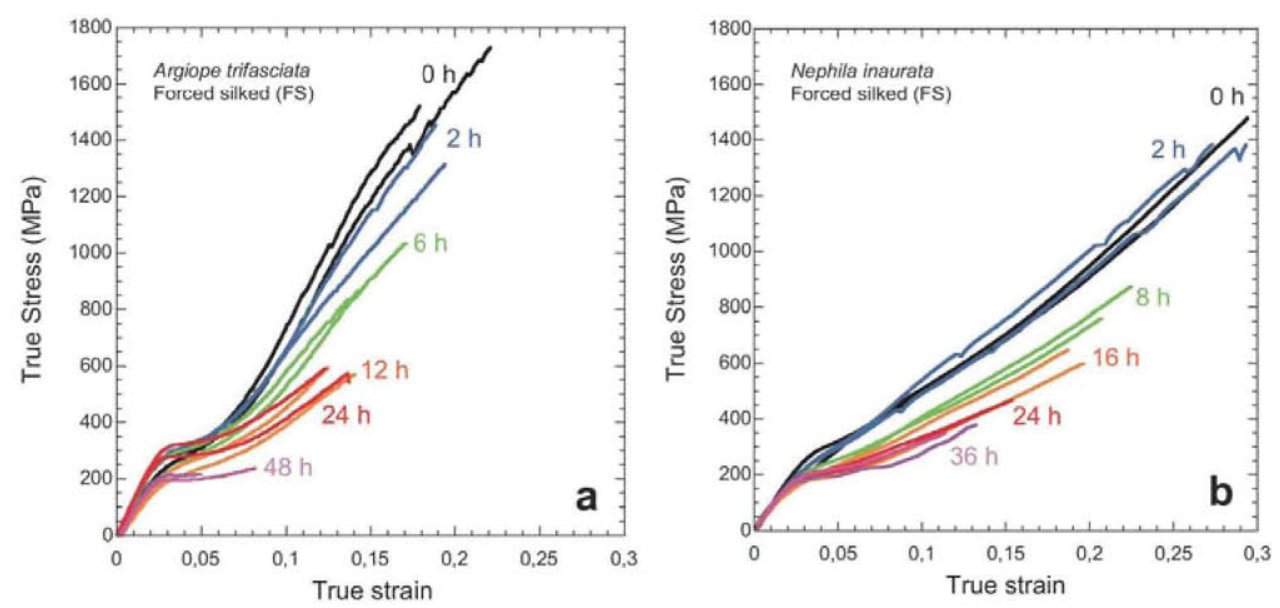

Fig. 1 True stress-true strain curves of MAS fibers from (a) Argiope trifasciata and (b) Nephila inaurata subjected to different UV irradiation times. 

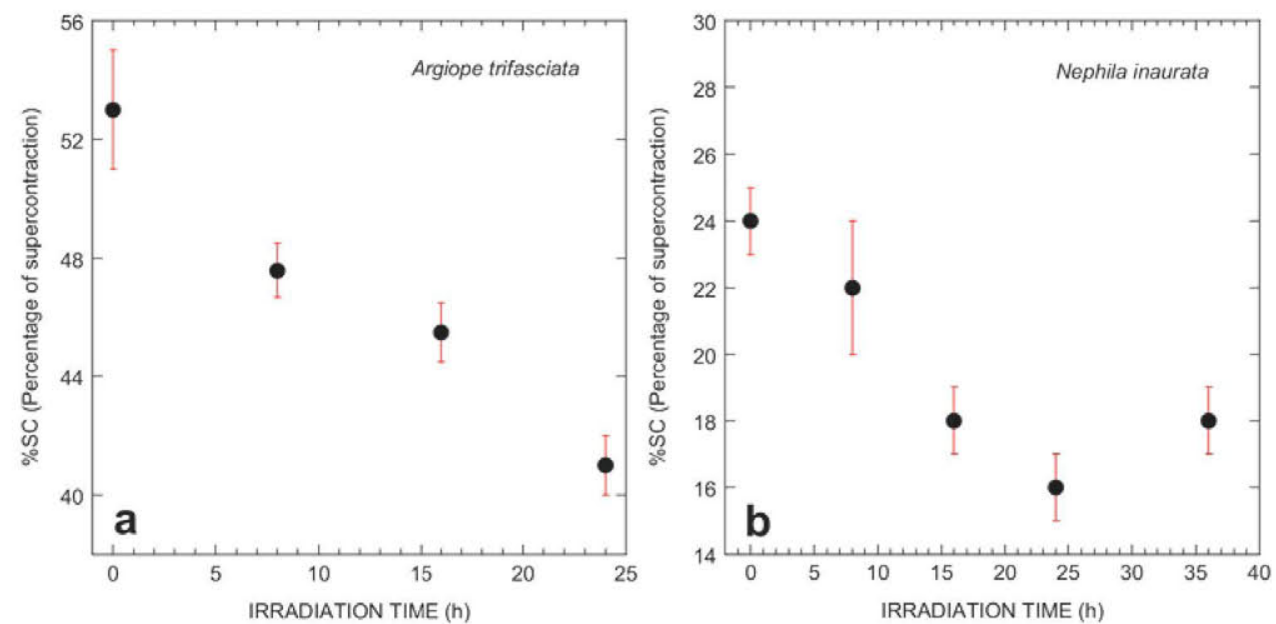

Fig. 3 Evolution of the percentage of supercontraction with UV irradiation time of (a) Argiope trifasciata and (b) Nephila inaurata MAS fibers subjected to maximum supercontraction after irradiation.

consistent with previous results which showed that the supercontraction effect presents significant quantitative variations among different spider species. ${ }^{14}$

The analysis of the tensile properties of - initially - UV irradiated samples subsequently subjected to maximum supercontraction shows that, although the percentage of supercontraction differs from that of control samples, no difference in the stress-strain curves of treated and control samples is observed up to the breaking point. This general behaviour is observed both when the supercontracted fibers are tested in air after drying (Fig. 4a) and in water (Fig. 4b).

\section{B. Biochemical analysis of UV irradiated fibers}

Following previous studies on silks and other polymers, it can be assumed that UV irradiation might lead either to the cleavage of the proteins, ${ }^{30}$ or to the formation of crosslinks, ${ }^{25}$ or to a combination of both mechanisms. In any case, cleavage and crosslinking imply a variation in the size of the proteins, so that some general trends between molecular weight and mechanical behaviour can be established from the analysis of UV irradiated samples independently from the specific molecular effect of UV light on silk. It is obvious, nevertheless, that the detailed identification of the molecular mechanism would allow establishing even deeper correlations between the microstructure and the properties of silk fibers.

To study the effect of UV radiation on MAS silk at a molecular level, we firstly analysed the effect of the radiation on the molecular weight of the silk proteins by SDS-polyacrylamide gel electrophoresis (SDS-PAGE). Fig. 5a shows the protein profile of samples retrieved by conventional forced silking after irradiation at different times from 0 (control) to 30 minutes. Protein bands are clearly observed only at very short irradiation times
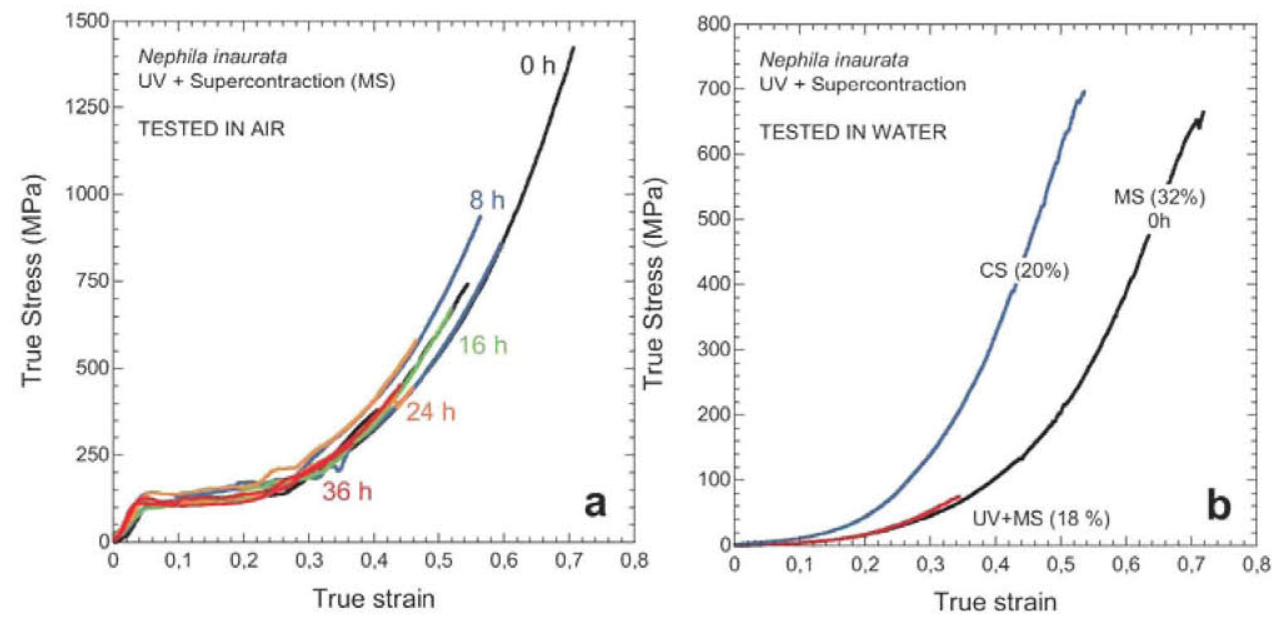

Fig. 4 True stress-true strain curves of Nephila inaurata MAS fibers subjected to UV irradiation and subsequent maximum supercontraction. (a) Fibers dried overnight after being subjected to maximum supercontraction and tested in air. (b) Fibers kept immersed in water after being subjected to maximum supercontraction and tested in water. MS states for maximum supercontraction, CS for controlled supercontraction (i.e. the fiber is allowed to supercontract but not to reach its maximum supercontracted length) and UV + MS for UV irradiation and subsequent maximum supercontraction. The number in parenthesis indicates the percentage of supercontraction $(\% \mathrm{SC})$ of each sample. 

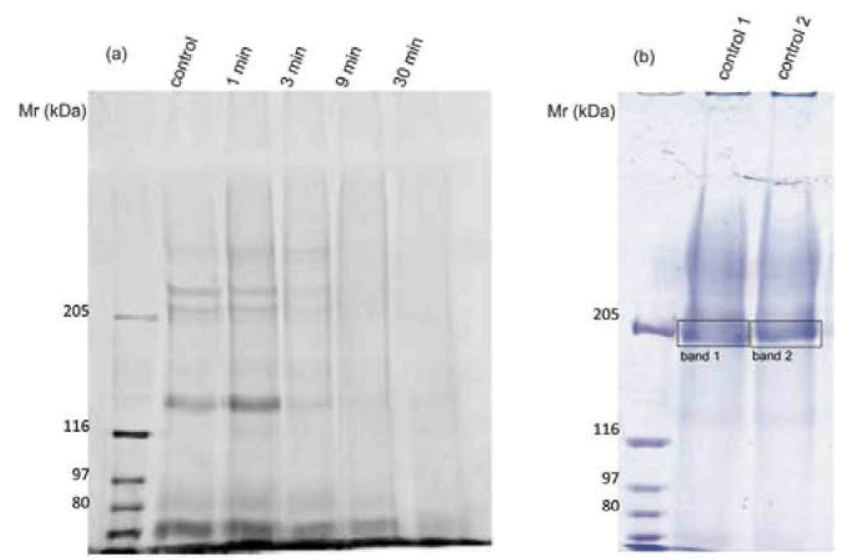

Fig. 5 SDS-PAGE analysis of Argiope trifasciata MAS spider silk. (a) Fibers were retrieved by massive forced silking extraction and subjected to different UV irradiation times. Irradiation times from 0 minutes (Control) to 30 minutes are indicated on each lane. (b) Fibers retrieved by monitored forced silking. Two replicates of control silk were used for protein identification by mass spectrometry. Molecular weight markers are shown on the left lane SDS-PAGE was performed with $5 \%$ polyacrylamide resolving gel and stained with Sypro Ruby (a) and Coomassie brilliant blue (b).

(up to 3 minutes) and disappear at longer irradiation periods. Three bands could be clearly identified in the gels, one intense band above the $116 \mathrm{kDa}$ marker, and two fainter bands above the $205 \mathrm{kDa}$ marker. As indicated above, both (1) the presence of an insoluble silk fraction in HFIP at long irradiation times and (2) the partial dissolution of the initially HFIP-dissolved and subsequently dried sample in the electrophoretic loading buffer required a careful assessment of the representativeness of the protein profiles with respect to the actual proteins in the fiber. Therefore, these bands were digested with chymotrypsin and subjected to LC-MS/MS analysis. The analysis revealed that the band at $116 \mathrm{kDa}$ (Fig. 5a) corresponded to acciniform silk, and those at $205 \mathrm{kDa}$ could be assigned to both MAS spidroin 1 and 2 proteins. ${ }^{3}$

The presence of proteins from acciniform silk in this preparation suggested that this type of silk could have been accidentally retrieved during the massive extraction of MAS silk with the conventional forced silking technique. Its appearance in the most intense band would simply reflect improved solubility in the electrophoretic loading buffer and, presumably, more efficient staining with Coomassie Brilliant Blue. This hypothesis was tested by analysing fibers obtained with the monitored forced silking, a process that minimizes the risk of contamination of MAS with other silk types. The corresponding gel is shown in Fig. $5 \mathrm{~b}$, where only the bands above the $205 \mathrm{kDa}$ marker were observed. These bands were again identified as MAS spidroin 1 and 2 using mass spectrometry (see ESI $\dagger$ ).

Fig. 5 a shows that all bands, including those corresponding to MAS spidroin 1 and 2, follow a similar trend with irradiation time, and disappear at relatively short ( 9 minutes) irradiation times. The information obtained from gel electrophoresis on its own, however, does not allow discriminating whether the disappearance of the bands is the result of protein cleavage or crosslinking. Thus, unspecific protein cleavage would lead to a set of shorter fragments that would not yield discrete bands in the gel, whereas crosslinking of proteins of molecular weights in excess of $200 \mathrm{kDa}^{5}$ would yield macromolecules that are not dissolved in SDS-buffer or cannot enter the gel. Two alternative strategies were followed to distinguish between cleavage and crosslinking: (1) a quantitative amino acid analysis of the HFIPinsoluble fraction in order to determine the ratio between the soluble and insoluble fractions at different irradiation times and (2) a repetition of the analysis performed on UV irradiated MAS silk with shorter recombinant proteins bioinspired in spider silk. ${ }^{45}$

The analysis of the insoluble/soluble fraction of silk from A. trifasciata and $N$. inaurata in HFIP with UV irradiation time is shown in Fig. 6. Note that the procedure used for separating both fractions (i.e. suction through a $300 \mu \mathrm{m}$ inner diameter needle) implies that the values found represent the lower limit for the insoluble fraction, since small portions of the insoluble fraction might be taken up and counted as soluble fraction. Formation of an insoluble material in principle might be favoured by longer irradiation times, which lead to more brittle fibers and increase the possibility of breaking small portions of insoluble fractions, and by the smaller diameter of the fibers. Both trends are observed when the results on UV irradiated fibers of $A$. trifasciata and $N$. inaurata (and recombinant proteins, see below) are compared.

As is observed in Fig. $6 a$ and b, the insoluble fraction represents the larger fraction at irradiation times up to 3-12 hours and reaches a maximum value of over $70 \%$ in $A$. trifasciata and slightly lower in $N$. inaurata. Increasing irradiation times then
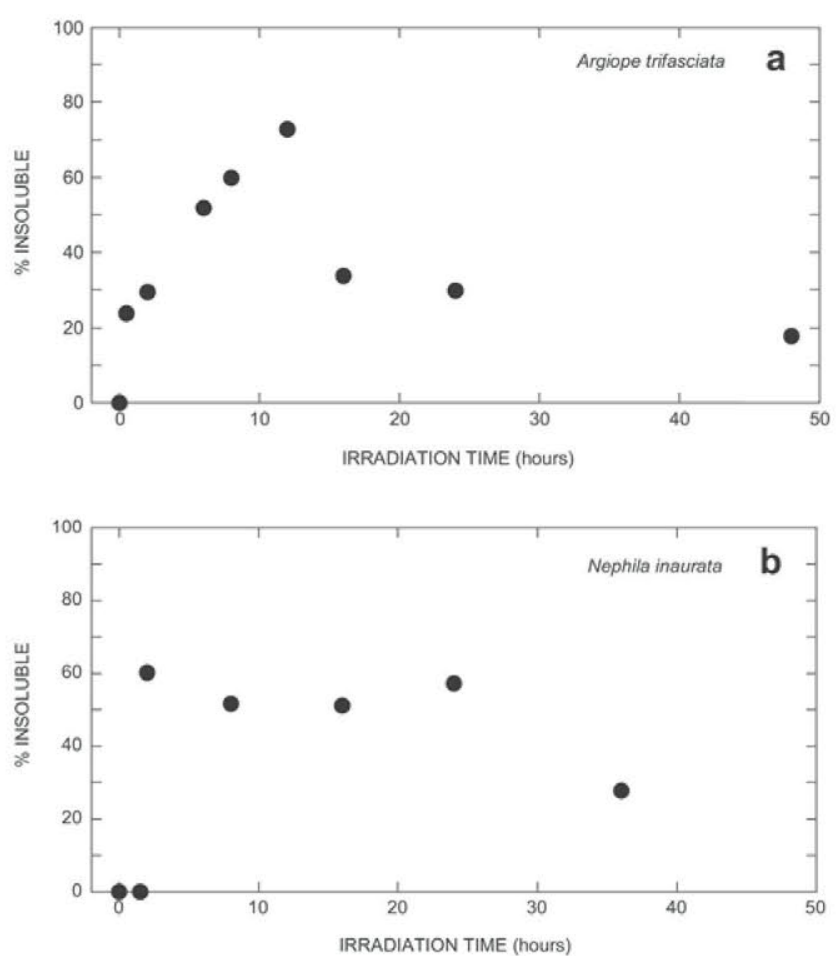

Fig. 6 Evolution of the insoluble fraction of silk with UV irradiation times of Argiope trifasciata (a) and Nephila inaurata (b) MAS fibers. 
leads to a decrease in the insoluble fraction, but it should be noticed, as commented above, that these results represent the lower limit of the content of the insoluble fraction in the sample, so that the actual value might be higher. No significant variation in the proportion of the amino acids is found either between the soluble and insoluble fractions or between control and irradiated samples, except for a steady decrease in the content of tyrosine with irradiation time from an initial value of $4 \%$ to a final value of $2.5 \%$ of the total mass. The results obtained from the analysis of the influence of irradiated recombinant protein fibers and from the quantitative amino acid analysis of natural silks suggest that crosslinking is, at least at these irradiation times, an effect exerted by exposure to UV light on these materials.

This assertion is further supported by the analysis of the effect of UV light on recombinant protein fibers as shown in Fig. 7. Recombinant bioinspired silk fibers with a composition based on spidroin 1 protein, but with a lower molecular weight of approx. $55 \mathrm{kDa}$, were UV irradiated. The larger cross-sectional area of the recombinant fibers (diameter $\sim 40 \mu \mathrm{m}$ ) compared with that of the natural material was taken into account by increasing UV exposure times. As observed in Fig. 7a, the effect of irradiation on the tensile behaviour of the recombinant fibers is similar to that observed in natural silk, since the true stresstrue strain curves of irradiated and control fibers concur up to the breaking of the former. In addition, the tensile strength and strain at breaking decrease in the recombinant protein fibers with increasing irradiation times.

An insoluble fraction was also found in recombinant protein fibers upon irradiation (Fig. 7b). Comparison with the results presented in Fig. 6 reveals that both natural and recombinant protein silks follow similar trends with an increase of the insoluble fraction at short irradiation times $(62 \%$ of the insoluble fraction after $2 \mathrm{~h}$ of UV irradiation in recombinant protein fibers), followed by a decrease of the insoluble fraction at longer irradiation times. The analysis by SDS-PAGE of the soluble fraction as presented in Fig. 7c shows a steady decrease in the intensity of the band at $55 \mathrm{kDa}$, corresponding to the recombinant protein as confirmed by mass spectrometry, until it finally disappears at an irradiation time of $8 \mathrm{~h}$. This behaviour parallels that previously observed in the natural proteins after irradiation as observed from Fig. 5.

However, in this case a faint band is observed at approximately $110 \mathrm{kDa}$, reaching a maximum of intensity at 30 minutes irradiation time and disappearing at $3 \mathrm{~h}$. Besides, several bands at the border between the stacking and resolving gels (i.e. in the limit of very high molecular weights) could be clearly observed in the gel for irradiation times between $30 \mathrm{~min}$ and 3 hours. The presence of these bands corresponding to high molecular weight moieties can be explained as the result of a crosslinking process between individual proteins. Crosslinking of just two reSp1 proteins leads to a $110 \mathrm{kDa}$ macromolecule compatible with the faint band observed in Fig. 7c. Crosslinks between a larger number of proteins would lead to moieties with increasing values of molecular weight. Thus, crosslinking between four or more reSp1 proteins would lead to moieties of over $200 \mathrm{kDa}$ that cannot enter the resolving gel and, consequently, accumulate at the border between stacking and resolving gels.
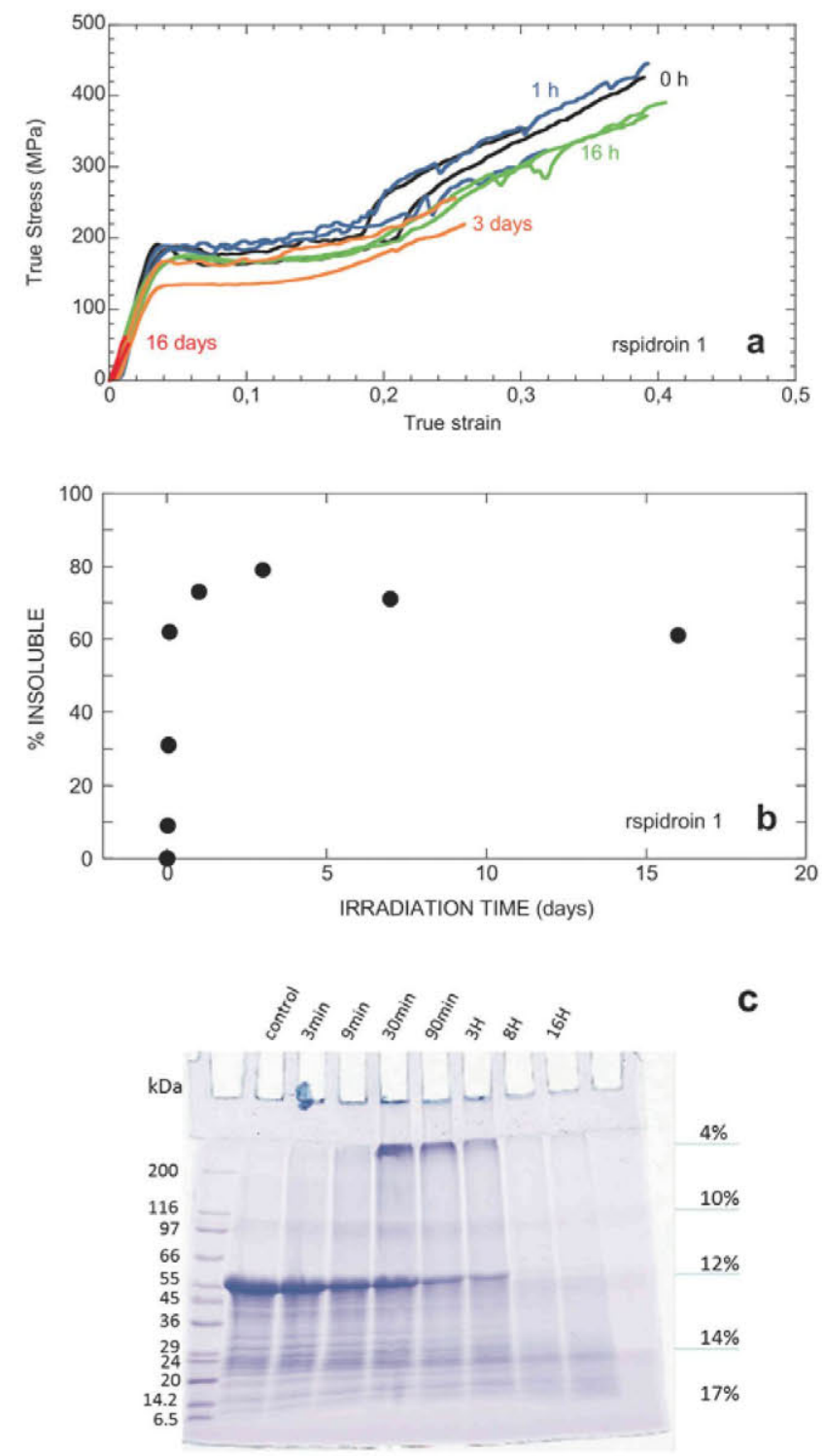

Fig. 7 (a) True stress-true strain curves of as-spun recombinant silk fibers subjected to different UV irradiation times. (b) Evolution of the insoluble fraction of recombinant silk with UV irradiation time. (c) Discontinuous gradient gel electrophoresis of recombinant spidroin fibers after different UV irradiation times. The original protein with a molecular weight of approx. $55 \mathrm{kDa}$ disappears gradually with irradiation time. Higher molecular weight products are observed at 30 minutes, 90 minutes and 3 hours. These products are considered to be the result of crosslinking the initial artificial spidroins

The presence of these bands confirms the existence of a process of UV-induced crosslinking between the protein fibers. Additional crosslinking, producing a further increase in molecular weight at longer irradiation times would then prevent the new moieties from even entering the gel, explaining the disappearance of the high molecular weight bands at irradiation times in excess of 3 hours, and the generation of the insoluble material. A similar mechanism could explain the absence of high molecular weight bands in the electrophoresis gels of the natural silks, since crosslinking from two individual 
proteins $\left(M_{\mathrm{W}} \sim 200 \mathrm{kDa}\right)$ would yield a macromolecule with a molecular weight of $M_{\mathrm{W}} \sim 400 \mathrm{kDa}$, which is too large to enter the gel.

As can be observed from the comparison of Fig. 7a and c, and Fig. 1 and 5, the time scale of the changes at a molecular level and their effects on the mechanical behaviour of the fibers occur at two different time scales. Thus, a significant modification of the proteins is observed in the range of a few minutes (natural spider silk) or a few hours (recombinant protein), while measurable changes in the tensile properties of the fibers are only observable at a time scale of hours (natural spider silk) and days (recombinant protein). These differences probably reflect that the chemical modification of the proteins sets in immediately upon starting irradiation, but there exists a threshold in the number of modifications until the mechanical behaviour of the fibers is affected. For instance, a single crosslink between two native proteins leads to a moiety of over $400 \mathrm{kDa}$ that cannot enter the gel, but it is not likely that a single additional covalent bond between two such large proteins can be measurable at a macroscopic level.

\section{Discussion}

The mechanical behaviour of UV irradiated samples reveals a number of interesting features on the relationship between the microstructure and the properties of spider silks, some of which are consistent with the usual models used to explain these materials, while others should be considered as unexpected or even anomalous in this framework. In this regard, the evolution of the stress-strain curves of FS fibers with irradiation time can be accounted for with the usual models by simply considering that the cleavage of proteins plays a leading role in determining the properties of the material. Protein cleavage would reduce the effective interaction length between proteins, so that the shorter peptides would transfer loads less efficiently to one another, which would explain the increased compliance of irradiated fibers. In addition, less efficient protein interactions would also imply the appearance of stress concentration sites where the contact between the protein chains is maintained. This effect is compatible with the decrease in the tensile strength and strain at breaking of the treated fibers. Unfortunately, this basic model cannot account for the mechanical behavior of either MS + UV or UV + MS samples.

With regard to the MS + UV fibers, the assumption of protein cleavage explains straightforwardly the reduced tensile strength and strain at breaking as discussed above for FS fibers. However, this simple model does not allow explaining the absence of the effect of UV irradiation on the overall stiffness of the MS fibers subjected to different irradiation times. In this regard, it is useful to consider that the deformation of spider silk fibers proceeds in three steps: ${ }^{47}$ initial deformation of hydrogen bonds, conformational changes in the protein chains from their initial configuration that allows the rotation of the $\beta$-nanocrystals, and stretching of the protein chains. The absence of a measurable effect on the tensile properties of irradiated samples supports that the third step results from the stretching of hidden length regions. ${ }^{48}$ These hidden length regions would be protein domains that are able to unfold, allowing a significant stretching of the chains from their initial apparent length. In this model, any modification at the molecular scale in the hidden length regions would remain unnoticed at low strain, i.e. before the chains begin to unfold. Fibers would break before the changes in the hidden length could be measured due to the inefficient load transfer and stress concentration in the most affected regions.

Finally, the justification of the tensile properties exhibited by UV irradiated and subsequently maximum supercontracted (UV + MS) samples represents another major challenge to present spider silk models. In this context, the decrease in \%SC with UV irradiation can be explained from a simple model by assuming that the cleavage of the proteins reduces the supercontraction stresses that build up in the fiber as a consequence of water immersion. ${ }^{49}$ Supercontraction stresses appear as the result of the collapse of the initial hydrogen bond lattice between protein chains in the fiber, ${ }^{20}$ whose tensile properties are then controlled by the elastomeric behaviour of the chains. ${ }^{50}$ Consequently, supercontraction relaxes the stresses in the protein chains by reducing the length of the fiber until the maximum supercontraction state is reached. In this regard, the decrease in the molecular weight of cleaved proteins would imply a reduction in the stress during supercontraction and, consequently, a smaller reduction in the length of the fiber would suffice to relax them.

However, as it happened with the MS + UV samples, this simple model cannot account for the concurrence of the tensile properties of treated and control samples up to the breaking point of the former. This concurrence is unexpected, since different values of the percentage of supercontraction lead to distinct tensile properties in non-irradiated samples, a property known as controlled supercontraction. ${ }^{21}$ This effect is evident in Fig. 4b, where the stress-strain curves of a maximum supercontracted control sample (MS; \%SC $=32 \%$ ), a sample subjected to $\mathrm{UV}$ irradiation for $36 \mathrm{~h}$ and supercontraction (UV $+\mathrm{MS} ; \% \mathrm{SC}=18 \%$ ) and a control sample subjected to controlled supercontraction $(\mathrm{CS} ; \% \mathrm{SC}=20 \%)$ are compared. As shown in the figure, the curves of the control maximum supercontracted and the irradiated and maximum supercontracted samples did concur up to the breaking point, and differ from that of the controlled supercontracted sample, despite the similar values of \%SC of the latter (UV + MS and CS) samples.

An explanation for this unexpected behaviour can be provided again by considering the existence of hidden length regions in the proteins. Since supercontraction depends on the ability of the chains to fold, the presence of hindrances that limit the folding ability of the proteins must affect the supercontraction process. However, supercontraction is a local phenomenon, ${ }^{51}$ so that UV irradiated and supercontracted fibers can be thought of as being composed of regions affected by UV radiation and those that essentially preserve their original properties. If the proteins in some of the hidden length regions undergo cleavage, the regions not so affected will still maintain their ability to supercontract and, consequently, will control the mechanical behaviour of UV + MS fibers at low and moderate strain. Thus, the stress-strain 
curves of the irradiated fibers would concur with those of control MS samples up to the breaking of the former, despite the fact that both irradiated and MS samples differ in the percentage of supercontraction. In addition, the extension of these regions would increase with longer UV exposure, which justifies the observed decrease of the percentage of supercontraction at increasing irradiation times.

Although relating the hidden length regions with the sequence of the spidroins at this point is necessarily speculative, the likely candidates to account for these regions are the -GGX- and -GPG- motifs. The presence of the -GGX- motif was related to the phenomenon of supercontraction itself $f^{12,52}$ while the -GPG- motif was related to the quantitative value of the shrinkage observed during supercontraction. ${ }^{13}$ Consequently, it is reasonable to assume that changes in any or both of these motifs at a molecular level may exert a significant influence on the mechanical behaviour of the fibers.

The hypothesis of an extensive cleavage of proteins caused by UV-light exposure is used above to justify the effects of irradiation on spider silk fibers as the result of an impaired Ioad transfer between the cleaved chains. The biochemical analysis, however, shows the presence - even possibly the prevalence - of extensive UV-induced protein crosslinking in the fibers. The consideration of the effects of UV radiation as the result of crosslinking between the proteins immediately highlights the role of the conformational freedom of the chains in the tensile behaviour of spider silk.

In this regard, the consideration of a restricted conformational freedom in UV-irradiated fibers combined with the concept of hidden length regions allows justifying the experimental results presented above:

- The reduced values of the tensile strength and strain at breaking found in all samples (FS, MS + UV and UV + MS) is explained by the creation of points of stress concentration at the crosslinks that promote the breaking of the chains.

- The reduced percentage of supercontraction can be explained by assuming that crosslinking in given regions would prevent the conformational changes in the chains which are required for supercontraction.

- The concurrence of the stress-strain curves of the MS + UV and UV + MS samples with those of the MS samples is explained by considering that the conformational restrictions established in the hidden length regions by crosslinking are not observed at low or moderate strain. At large strain stress concentration leads to the breaking of the fibers. In this context, crosslinks established between the alanine residues that form the $\beta$-nanocrystals, if present, are not likely to modify significantly the stiff mechanical behaviour of the crystalline phase.

- Finally, the decrease in stiffness observed in FS fibers exposed to UV radiation can be explained by assuming that reaching the FS properties from the MS state requires enough conformational freedom of the proteins to allow increasing the alignment of the chains. If the conformational freedom is restricted, the final stress-strain curve could be some intermediate state between that of the initial largely coiled MS state and that of the fully stretched FS state. Experimentally, this would appear as an increase in the compliance of the fibers with increasing irradiation time, consistent with the experimental observations. Alternatively, a random combination of cleavage and crosslinking can also lead to an increase of compliance in systems formed from highly aligned chains, if crosslinking is supposed to be established in the transverse direction of the fibers and cleavage occurs along the longitudinal direction of the chains.

\section{Conclusions}

The analysis of the results presented in this work allows relating the tensile properties of silk fibers with molecular aspects of their building elements, so that new insights into the relationship between the microstructure and the properties of this material are gained. It is observed that exposure to UV light for increasing irradiation times leads to a steady decrease of the tensile properties of silk both in terms of tensile strength and strain at breaking. A detailed study of the changes in the mechanical behaviour of MAS fibers either directly retrieved by forced silking or subjected to a previous supercontraction step suggests the relationship of their high performance and the conformational freedom of its constituent spidroins. This idea is significantly reinforced when the mechanical characterization is extended to include the effect of supercontraction on previously UV irradiated samples.

The biochemical analysis revealed that extensive crosslinking between proteins results after exposure to UV light, conferring a significant - if not prevalent - role to this mechanism in determining the mechanical behaviour of the irradiated fibers. The negative effect of these crosslinks on the mechanical performance of the fiber stresses the importance of preventing the creation of entanglements between the chains that would limit their conformational freedom.

The suggestive conclusion could be, finally, that Nature seems to have developed a strategy that maximizes conformational freedom in silk fibers despite being made up of singularly long macromolecules. This would be the result, in turn, of minimizing the number of entanglements between the chains through the adequate topological organization of the chains.

\section{Authors contributions}

GBP and JMPM performed the mechanical characterization. CS performed the biochemical analysis with the help of IJ and JV. JPR coordinated the research and wrote the main text. All authors contributed to the data analysis and reviewed the manuscript.

\section{Acknowledgements}

Spiders were reared in Reptilmadrid S.L. by Oscar Campos. Nexia fibers were kindly provided by Prof. Costas Karatzas (McGill University, Canada). The authors are grateful to José Miguel Martínez for his help with the artwork. The work was funded by Ministerio de Economía y Competitividad (Spain) 
through project MAT2012-38412-C02-01, by the Comunidad de Madrid (Spain) (Grant S2011/BMD-2460), by Fundación Marcelino Botín and by Banco Santander through its Santander University Global Division.

\section{References}

1 M. Heim, D. Keerl and T. Scheibel, Angew. Chem., Int. Ed., 2009, 48, 3584-3596.

2 D. Porter and F. Vollrath, Adv. Mater, 2009, 21, 487-492, DOI: $10.1002 /$ adma.200801332 ER.

3 J. Gatesy, C. Hayashi, D. Motriuk, J. Woods and R. Lewis, Science, 2001, 291, 2603-2605.

4 F. Vollrath, Sci. Am., 1992, 266, 70-76.

5 M. Xu and R. V. Lewis, Proc. Natl. Acad. Sci. U. S. A., 1990, 87, 7120-7124.

6 T. A. Blackledge, N. Scharff, J. A. Coddington, T. Szuts, J. W. Wenzel, C. Y. Hayashi and I. Agnarsson, Proc. Natl. Acad. Sci. U. S. A., 2009, 106, 5229-5234, DOI: 10.1073/ pnas.0901377106 ER.

7 G. V. Guinea, M. Elices, G. R. Plaza, G. B. Perea, R. Daza, C. Riekel, F. Agullo-Rueda, C. Hayashi, Y. Zhao and J. PerezRigueiro, Biomacromolecules, 2012, 13, 2087-2098, DOI: 10.1021/bm3004644.

8 F. Vollrath and D. T. Edmonds, Nature, 1989, 340, 305-307.

9 T. A. Blackledge and C. Y. Hayashi, J. Exp. Biol., 2006, 209, 2452-2461, DOI: 10.1242/jeb.02275 ER.

10 T. A. Blackledge, J. Pérez-Rigueiro, G. R. Plaza, B. Perea, A. Navarro, G. V. Guinea and M. Elices, Sci. Rep., 2012, 2, 782, DOI: 10.1038/srep00782.

11 R. W. Work, Text. Res. J., 1977, 47, 650-662.

12 J. Pérez-Rigueiro, G. R. Plaza, F. G. Torres, A. Hijar, C. Hayashi, G. B. Perea, M. Elices and G. V. Guinea, Int. J. Biol. Macromol., 2010, 46, 555-557.

13 Y. Liu, A. Sponner, D. Porter and F. Vollrath, Biomacromolecules, 2008, 9, 116-121, DOI: 10.1021/bm700877g ER.

14 C. Boutry and T. A. Blackledge, J. Exp. Biol., 2010, 213, 3505-3514, DOI: 10.1242/jeb.046110.

15 M. Tsukada, M. Nagura, H. Ishikawa and H. Shiozaki, J. Appl. Polym. Sci., 1991, 43, 643-649, DOI: 10.1002/app.1991.070430403.

16 H. Somashekarappa, N. Selvakumar, v. Subramaniam and R. Somashekar, J. Appl. Polym. Sci., 1996, 59, 1677-1681, DOI: $10.1002 /($ SICI)1097-4628(19960314)59:11<1677::AIDAPP3 > 3.0.CO;2-L.

17 D. L. Dunaway, B. L. Thiel and C. Viney, J. Appl. Polym. Sci., 1995, 58, 675-683.

18 A. Martel, M. Burghammer, R. J. Davies and C. Riekel, Biomacromolecules, 2007, 8, 3548-3556, DOI: 10.1021/ bm700935w ER.

19 Z. Z. Shao and F. Vollrath, Polymer, 1999, 40, 1799-1806.

20 Y. Termonia, Macromolecules, 1994, 27, 7378-7381.

21 J. Perez-Rigueiro, M. Elices and G. V. Guinea, Polymer, 2003, 44, 3733-3736, DOI: 10.1016/S0032-3861(03)00245-3 ER.

22 J. Guan, F. Vollrath and D. Porter, Biomacromolecules, 2011, 12, 4030-4035, DOI: $10.1021 / \mathrm{bm} 201032 \mathrm{v}$.
23 M. A. Becker, P. Willman and N. C. Tuross, J. Am. Inst. Conserv., 1995, 34, 141-152, DOI: $10.2307 / 3179736$.

24 S. Baltova and V. Vassileva, Polym. Degrad. Stab., 1998, 60, 61-65, DOI: 10.1016/S0141-3910(98)80027-X.

25 A. Sionkowska and A. Planecka, Polym. Degrad. Stab., 2011, 96, 523-528, DOI: 10.1016/j.polymdegradstab.2011.01.001.

26 C. Solazzo, J. M. Dyer, S. Deb-Choudhury, S. Clerens and P. Wyeth, Photochem. Photobiol., 2012, 88, 1217-1226, DOI: 10.1111/j.1751-1097.2012.01167.x.

27 S. Osaki, Polym. J., 2004, 36, 657-660, DOI: 10.1295/polymj.36.657.

28 J. Perez-Rigueiro, M. Elices, G. R. Plaza, J. Rueda and G. V. Guinea, J. Polym. Sci., Part B: Polym. Phys., 2007, 45, 786-793, DOI: 10.1002/polb.21118 ER.

29 S. Osaki, K. Yamamoto, A. Kajiwara and M. Murata, Polym. J., 2004, 36, 623-627, DOI: 10.1295/polymj.36.623.

30 T. Matsuhira, K. Yamamoto and S. Osaki, Polym. J., 2013, 45, 1167-1169, DOI: 10.1038/pj.2013.41.

31 I. Marshall and A. Todd, Trans. Faraday Soc., 1953, 49, 67-78, DOI: 10.1039/tf9534900067.

32 S. Tsuge, H. Yokoi, Y. Ishida, H. Ohtani and M. A. Becker, Polym. Degrad. Stab., 2000, 69, 223-227, DOI: 10.1016/S01413910(00)00067-7.

33 R. W. Work and P. D. Emerson, J. Arachnol., 1982, 10, 1-10. 34 J. Perez-Rigueiro, M. Elices, G. Plaza, J. I. Real and G. V. Guinea, J. Exp. Biol., 2005, 208, 2633-2639, DOI: 10.1242/jeb.01701 ER.

35 J. Perez-Rigueiro, C. Viney, J. Llorca and M. Elices, J. Appl. Polym. Sci., 1998, 70, 2439-2447.

36 G. V. Guinea, M. Elices, J. I. Real, S. Gutierrez and J. PerezRigueiro, J. Exp. Zool., Part A, 2005, 303A, 37-44, DOI: 10.1002/jez.a.111 ER.

37 G. V. Guinea, M. Elices, J. Perez-Rigueiro and G. R. Plaza, J. Exp. Biol., 2005, 208, 25-30.

$38 \mathrm{~J}$. Perez-Riguero, M. Elices, J. Llorca and C. Viney, J. Appl. Polym. Sci., 2001, 82, 2245-2251.

39 G. V. Guinea, J. Perez-Rigueiro, G. R. Plaza and M. Elices, Biomacromolecules, 2006, 7, 2173-2177, DOI: 10.1021/ bm060138v ER.

40 U. K. Laemmli, Nature, 1970, 227, 680-685, DOI: 10.1038/ $227680 \mathrm{a} 0$.

41 I. Jorge, E. M. Casas, M. Villar, I. Ortega-Perez, D. LopezFerrer, A. Martinez-Ruiz, M. Carrera, A. Marina, P. Martinez, H. Serrano, B. Canas, F. Were, J. M. Gallardo, S. Lamas, J. M. Redondo, D. Garcia-Dorado and J. Vazquez, J. Mass Spectrom., 2007, 42, 1391-1403, DOI: 10.1002/jms.1314.

42 D. Lopez-Ferrer, S. Martinez-Bartolome, M. Villar, M. Campillos, F. Martin-Maroto and J. Vazquez, Anal. Chem., 2004, 76, 6853-6860, DOI: 10.1021/ac049305c.

43 P. Navarro and J. Vazquez, J. Proteome Res., 2009, 8, 1792-1796, DOI: $10.1021 / \mathrm{pr} 800362 \mathrm{~h}$.

44 S. Martinez-Bartolome, P. Navarro, F. Martin-Maroto, D. LopezFerrer, A. Ramos-Fernandez, M. Villar, J. P. Garcia-Ruiz and J. Vazquez, Mol. Cell. Proteomics, 2008, 7, 1135-1145, DOI: 10.1074/mcp.M700239-MCP200.

45 A. Lazaris, S. Arcidiacono, Y. Huang, J. F. Zhou, F. Duguay, N. Chretien, E. A. Welsh, J. W. Soares and C. N. Karatzas, Science, 2002, 295, 472-476. 
46 M. Elices, G. V. Guinea, G. R. Plaza, C. Karatzas, C. Riekel, F. Agullo-Rueda, R. Daza and J. Perez-Rigueiro, Macromolecules, 2011, 44, 1166-1176, DOI: 10.1021/ma102291m ER.

47 A. Tarakanova and M. J. Buehler, JOM, 2012, 64, 214-225, DOI: $10.1007 / \mathrm{s} 11837-012-0250-3$.

48 A. Nova, S. Keten, N. M. Pugno, A. Redaelli and M. J. Buehler, Nano Lett., 2010, 10, 2626-2634, DOI: 10.1021/nl101341w ER.
49 K. N. Savage, P. A. Guerette and J. M. Gosline, Biomacromolecules, 2004, 5, 675-679, DOI: 10.1021/bm034270w ER.

50 J. M. Gosline, M. W. Denny and M. E. Demont, Nature, 1984, 309, 551-552.

51 P. T. Eles and C. A. Michal, Macromolecules, 2004, 37, 1342-1345, DOI: 10.1021/ma035567p ER.

52 Z. T. Yang, O. Liivak, A. Seidel, G. LaVerde, D. B. Zax and L. W. Jelinski, J. Am. Chem. Soc., 2000, 122, 9019-9025. 